TAMKANG JOURNAL OF MATHEMATICS

Volume 33, Number 4, Winter 2002

\title{
ON ABSOLUTE NÖRLUND SUMMABILITY OF FOURIER SERIES
}

\author{
B. E. RHOADES AND EKREM SAVAŞ
}

\begin{abstract}
We obtain two theorems on the absolute Nörlund summability of Fourier series and factored Fourier series.
\end{abstract}

In 1990 Sulaiman [3] obtained sufficient conditions for a Fourier series and the derived Fourier seriese to be absolutely Nörlund summable of order $k \geq 1$. However, he used an incorrect definition of absolute summability. (See, e.g., [2].)

In this paper we obtain the comparable results of [3] using the correct definition.

Let $A$ be a lower triangular matrix, $\sum a_{k}$ a series with partial sums $s_{n}$,

$$
T_{n}:=\sum_{k=0}^{n} a_{n k} s_{k} .
$$

Then the series $\sum a_{k}$ is absolutely summable $A$ of order $k$, written $|A|_{k}$, if

$$
\sum_{n=1}^{\infty} n^{k-1}\left|T_{n}-T_{n-1}\right|^{k}<\infty
$$

Let $f$ be a $2 \pi$-periodic function, Lebesgue integrable over $(-\pi, \pi)$.

$$
f(x) \sim \frac{a_{0}}{2}+\sum_{n=1}^{\infty}\left(a_{n} \cos n x+b_{n} \sin n x\right):=\sum_{n=0}^{\infty} A_{n}(x) .
$$

The derived series for $f$ is denoted by

$$
\sum_{n=1}^{\infty} n B_{n}(x):=\sum_{n=1}^{\infty} n\left(b_{n} \cos n x-a_{n} \sin n x\right) .
$$

We shall write

$$
\begin{aligned}
\phi(u) & =f(x+u)+f(x-u)-2 f(x) \\
\psi(u) & =f(x+u)-f(x-u)-2 f^{\prime}(x) \\
\phi_{1}(u) & =\frac{1}{t} \int_{0}^{t} \phi(u) d u=\frac{1}{t} \Phi(t) \\
\psi_{1}(t) & =\frac{1}{t} \int_{0}^{t} d \psi(u) d u=\frac{1}{t} \psi_{2}(t) .
\end{aligned}
$$

Received October 30, 2001.

2000 Mathematics Subject Classification. 40F05, 40G99, 42A24.

Key words and phrases. Absolute summability, Nörlund, Fourier series. 
For any sequence $\left\{u_{n}\right\}, \Delta u_{n}:=u_{n}-u_{n+1}$ and $\nabla u_{n}:=u_{n}-u_{n-1}$. Let $h$ denote a positive function such that, for some $\beta, 0<\beta<1, u \beta h\left(u^{-1}\right)$ is nondecreasing.

A Nörlund matrix is a lower trianglular matrix with entries $p_{n-k} / P_{n}$, where $\left\{p_{k}\right\}$ is a positive sequence and $P_{n}:=\sum_{k=0}^{n} p_{k}$. The condition $\lim p_{n} / P_{n}=0$ is necessary and sufficient for $\left(N, p_{n}\right)$ to be regular.

We shall prove the following two theorems.

Theorem 1. Let $\left\{p_{n}\right\}$ be a positive sequence such that $\left\{\nabla p_{n}\right\}$ is bounded, nonincreasing, $\left\{1 / p_{n}^{k-1} P_{n}\right\}$ is nonincreasing, and

$$
\frac{n p_{n}}{P_{n}}=O(1)
$$

If

$$
\Phi_{1}(t):=\int_{t}^{\delta} u^{-1} \phi_{1}(u) d u=O\left(h\left(t^{-1}\right)\right)
$$

and

$$
\sum \frac{n^{k}[h(n)]^{k}}{p_{n}^{1-k} P_{n}}<\infty
$$

then the series (1) is summable $\left|N, p_{n}\right|_{k}, k \geq 1$.

Theorem 2. Let $\left\{p_{n}\right\}$ satisfy the conditions of Theorem 1. If

$$
\Psi(t):=\int_{t}^{\delta} u^{-1} \Psi_{1}(u) d u=O\left(h\left(t^{-1}\right)\right)
$$

then the series (2) is summable $\left|N, p_{n}\right|_{k}, k \geq 1$, provided (4) exists.

The proof of Theorem 1 requires the following lemma.

Lemma 1. Let $s_{n}^{(1)}:=\sum_{k=0}^{n} s_{k}$. If $\left\{p_{n}\right\}$ is a sequence satisfying $(3),\left\{\nabla p_{n}\right\}$ and $\left\{1 /\left(p_{n}^{k-1} P_{n}\right)\right\}$ are nonincreasing, and

$$
\sum_{n=0}^{\infty} \frac{\left|s_{n}^{(1)}\right|^{k}}{p_{n}^{k-1} P_{n}}<\infty
$$

then the series $\sum a_{k}$ is summable $\left|N, p_{n}\right|_{k}, k \geq 1$.

Proof. As in [3] we may write

$$
\begin{aligned}
t_{n-1}-t_{n}= & \Delta\left(\frac{1}{P_{n-1}}\right) \sum_{v=0}^{n-2} \nabla_{n}\left(p_{n-1-v}\right) s_{v}^{(1)}+\Delta\left(\frac{1}{P_{n-1}}\right) p_{0} s_{n-1}^{(1)} \\
& +\frac{1}{P_{n}} \sum_{v=0}^{n-2} \nabla_{n}\left(p_{n-1-v}-p_{n-v}\right) s_{v}^{(1)}+\frac{1}{P_{n}}\left(p_{0}-p_{1}\right) s_{n-1}^{(1)}-\frac{p_{0} s_{n}^{(1)}}{P_{n}}+\frac{p_{0} s_{n-1}^{(1)}}{P_{n}} \\
& =T_{1}+T_{2}+T_{3}+T_{4}+T_{5}+T_{6}, \quad \text { say. }
\end{aligned}
$$




$$
\begin{aligned}
\sum_{n=1}^{\infty} n^{k-1}\left|T_{1}\right|^{k} & =\sum_{n=1}^{\infty} n^{k-1}\left|\frac{p_{n}}{P_{n} P_{n-1}} \sum_{v=0}^{n-2} \nabla_{n}\left(p_{n-1-v}\right) s_{v}^{(1)}\right|^{k} \\
& \leq \sum_{n=1}^{\infty} n^{k-1} \frac{p_{n}^{k}}{P_{n}^{k} P_{n-1}} \sum_{v=0}^{n-2} p_{v}^{1-k}\left|\nabla_{n} p_{n-1-v}\right|^{k}\left|s_{v}^{(1)}\right|^{k}\left(\frac{1}{P_{n-1}} \sum_{v=0}^{n-2} p_{v}\right)^{k-1} \\
& =O(1) \sum_{n=1}^{\infty} \frac{n^{k-1} p_{n}^{k}}{P_{n}^{k} P_{n-1}} \sum_{v=0}^{n-2} p_{v}^{1-k}\left|s_{v}^{(1)}\right|^{k} \\
& =O(1) \sum_{v=0}^{\infty} p_{v}^{1-k}\left|s_{v}^{(1)}\right|^{k} \sum_{n=v+1}^{\infty} \frac{n^{k-1} p_{n}^{k}}{P_{n}^{k} P_{n-1}}
\end{aligned}
$$

Using (3),

$$
\sum_{n=v+1}^{\infty} \frac{n^{k-1} p_{n}^{k}}{P_{n}^{k} P_{n-1}}=O\left(\frac{1}{P_{v}}\right)
$$

Thus

$$
\sum_{n=1}^{\infty} n^{k-1}\left|T_{1}\right|^{k}=O(1) \sum_{v=0}^{\infty} p_{v}^{1-k}\left|s_{v}^{(1)}\right|^{k} \frac{1}{P_{v}}=O(1) .
$$

Using (3),

$$
\begin{aligned}
& \sum_{n=1}^{\infty} n^{k-1}\left|T_{2}\right|^{k}=\sum_{n=1}^{\infty} n^{k-1}\left|\Delta\left(\frac{1}{P_{n-1}}\right) p_{0} s_{n-1}^{(1)}\right|^{k} \\
& =\sum_{n=1}^{\infty} n^{k-1}\left(\frac{p_{n}}{P_{n} P_{n-1}}\right)^{k} p_{o}^{k}\left|s_{n-1}^{(1)}\right|^{k} \\
& =O(1) \sum_{n=1}^{\infty}\left(\frac{n p_{n}}{P_{n}}\right)^{k-1} \frac{p_{n}}{P_{n} P_{n-1}^{k}}\left|s_{n-1}^{(1)}\right|^{k} \\
& =O(1) \sum_{n=1}^{\infty} \frac{1}{P_{n-1}^{k}}\left|s_{n-1}^{(1)}\right|^{k} \\
& =O(1) \sum_{n=1}^{\infty}\left(\frac{p_{n-2}}{P_{n-2}}\right)^{k-1}\left(\frac{P_{n-2}}{P_{n-1}}\right)^{k-1} \frac{\left|s_{n-1}^{(1)}\right|^{k}}{p_{n-2}^{k-1} P_{n-1}} \\
& =O(1) \sum_{n=1}^{\infty} \frac{\left|s_{n-1}^{(1)}\right|^{k}}{p_{n-2}^{k-1} P_{n-1}}=O(1) \text {. } \\
& \sum_{n=1}^{\infty} n^{k-1}\left|T_{3}\right|^{k}=\sum_{n=1}^{\infty} n^{k-1}\left|\frac{1}{P_{n}} \sum_{v=0}^{n-2} \nabla_{n}\left(p_{n-1-v}-p_{n-v}\right) s_{v}^{(1)}\right|^{k} \\
& \leq \sum_{n=1}^{\infty} \frac{n^{k-1}}{P_{n}^{k}} \sum_{v=0}^{n-2}\left|\nabla_{n}\left(p_{n-1-v}-p_{n-v}\right)\right|\left|s_{v}^{(1)}\right|^{k}\left(\sum_{v=0}^{n-2}\left|\nabla_{n}\left(p_{n-1-v}-p_{n-v}\right)\right|\right)^{k-1}
\end{aligned}
$$




$$
\begin{aligned}
& =O(1) \sum_{n=1}^{\infty} \frac{n^{k-1}}{P_{n}^{k}} \sum_{v=0}^{n-2}\left|\nabla_{n}\left(p_{n-1-v}-p_{n-v}\right)\right|\left|s_{v}^{(1)}\right|^{k} \\
& =O(1) \sum_{b=0}^{\infty}\left|s_{v}^{(1)}\right|^{k} \sum_{v=1}^{\infty} \frac{n^{k-1}}{P_{n}^{k}}\left(\nabla_{n}\left(p_{n-1-v}-p_{n-v}\right)\right) .
\end{aligned}
$$

Using (3),

$$
\frac{n^{k-1}}{P_{n}^{k}}=\left(\frac{n p_{n}}{P_{n}}\right)^{k-1} \frac{1}{p_{n}^{k-1} P_{n}}=\frac{O(1)}{p_{n}^{k-1} P_{n}} .
$$

Therefore

$$
\begin{aligned}
& \sum_{n=1}^{\infty} n^{k-1}\left|T_{3}\right|^{k}=O(1) \sum_{v=0}^{\infty}\left|s_{v}^{(1)}\right|^{k} \sum_{n=v+1}^{\infty} \frac{1}{p_{n}^{k-1} P_{n}} \nabla_{n}\left(p_{n-1-v}-p_{n-v}\right) \\
& =O(1) \sum_{v=0}^{\infty} \frac{\left|s_{v}^{(1)}\right|^{k}}{p_{v+1}^{k-1} P_{v+1}} \sum_{n=v+1}^{\infty} \nabla_{n}\left(p_{n-1-v}-p_{n-v}\right) \\
& =O(1) \sum_{v=0}^{\infty} \frac{\left|s_{v}^{(1)}\right|^{k}}{p_{v}^{k-1} P_{v}}=O(1) \text {. } \\
& \sum_{n=1}^{\infty} n^{k-1}\left|T_{4}\right|^{k}=\sum_{n=1}^{\infty} n^{k-1}\left|\frac{1}{P_{n}}\left(p_{0}-p_{1}\right) s_{n-1}^{(1)}\right|^{k} \\
& =O(1) \sum_{n=1}^{\infty} \frac{n^{k-1}}{P_{n}^{k}}\left|s_{n-1}^{(1)}\right|^{k} \\
& =O(1) \sum_{n=1}^{\infty} \frac{\left|s_{n-1}^{(1)}\right|^{k}}{p_{n}^{k-1} P_{n}} \\
& =O(1) \sum_{n=1}^{\infty} \frac{\left|s_{n-1}^{(1)}\right|^{k}}{p_{n-1}^{k-1} P_{n-1}}=O(1) \text {. } \\
& \sum_{n=1}^{\infty} n^{k-1}\left|T_{5}\right|^{k}=\sum_{n=1}^{\infty} n^{k-1}\left|\frac{p_{0} s_{n}^{(1)}}{P_{n}}\right|^{k} \\
& =O(1) \sum_{n=1}^{\infty} \frac{n^{k-1}\left|s_{n}^{(1)}\right|^{k}}{P_{n}^{k}} \\
& =O(1) \sum_{n=1}^{\infty} \frac{\left|s_{n}^{(1)}\right|^{k}}{p_{n}^{k-1} P_{n}}=O(1) \text {. } \\
& \sum_{n=1}^{\infty} n^{k-1}\left|T_{6}\right|^{k}=\sum_{n=1}^{\infty} n^{k-1}\left|\frac{p_{0} s_{n-1}^{(1)}}{P_{n}}\right|^{k}
\end{aligned}
$$




$$
\begin{aligned}
& =O(1) \sum_{n=1}^{\infty} \frac{n^{k-1}\left|s_{n-1}^{(1)}\right|^{k}}{P_{n}^{k}} \\
& =O(1) \sum_{n=1}^{\infty} \frac{\left|s_{n-1}^{(1)}\right|^{k}}{p_{n}^{k-1} P_{n}} \\
& =O(1) \sum_{n=1}^{\infty} \frac{\left|s_{n-1}^{(1)}\right|^{k}}{p_{n-1}^{k-1} P_{n-1}}=O(1) .
\end{aligned}
$$

\section{Proof of Theorem 1.}

$$
\begin{aligned}
\sum_{v=0}^{n}\left[s_{v}(x)-f(x)\right] & =\frac{2}{\pi} \int_{0}^{\pi}\left\{t^{-1} \phi_{1}(t)+\phi_{1}^{\prime}(t)\right\} \sum_{v=0}^{n} \sin v t d t+o(n) \\
& =\frac{2}{\pi}\left\{I_{1}+I_{2}\right\}, \quad \text { say. } \\
I_{1} & =\int_{0}^{1 / n}+\int_{1 / n}^{\pi}=I_{11}+I_{12}, \quad \text { say. } \\
I_{11} & =\int_{0}^{1 / n} t^{-1} \phi_{1}(t) \sum_{v=0}^{n} \sin v t d t \\
& =O\left(n^{2}\right) \int_{0}^{1 / n} t^{-1} \phi_{1}(t) d t=O(n h(n))
\end{aligned}
$$

since, as shown in [3],

$$
\begin{aligned}
\Phi_{1}(t)=O\left\{h\left(t^{-1}\right)\right\} \quad \text { implies that } \quad \int_{0}^{t} \phi_{1}(u) d u=O\left\{\operatorname{th}\left(t^{-1}\right)\right\} . \\
I_{12}=O(n) \int_{1 / n}^{\pi} t^{-1} \phi_{1}(t) d t=O(n h(n)) . \\
I_{2}=\int_{0}^{\pi} \phi_{1}^{\prime}(t) \sum_{v=0}^{n} \sin v t d t \\
=-\left(\int_{0}^{1 / n}+\int_{1 / n}^{\pi}\right) \phi_{1}(t) \sum_{v=0}^{n} v \cos v t d t \\
=-\left(I_{21}+I_{22}\right), \quad \operatorname{say.} \\
I_{21}=O\left(n^{2}\right) \int_{0}^{1 / n} \phi_{1}(t) d t=O(n h(n)) . \\
I_{22}=O(1) \int_{1 / n}^{\pi} \phi_{1}(t)\left|\sum_{v=0}^{n} v \cos v t\right| d t
\end{aligned}
$$




$$
\begin{aligned}
& =O(n) \int_{1 / n}^{\pi} \phi_{1}(t) \max _{0 \leq r \leq 1}\left|\sum_{v=0}^{n} \cos v t\right| d t \\
& =O(n) \int_{1 / n}^{\pi} t^{-1} \phi_{1}(t) d t=O(n h(n)) .
\end{aligned}
$$

Therefore

$$
\begin{aligned}
& \sum_{v=0}^{n}\left[s_{v}(x)-f(x)\right]=O\{n h(n)\} \\
&\left|\sum_{v=0}^{n} s_{v}(x)\right| \leq\left|\sum_{v=0}^{n}\left[s_{v}(x)-f(x)\right]\right|+\left|\sum_{v=0}^{n} f(x)\right| \\
&=O\{n h(n)\} .
\end{aligned}
$$

The proof of Theorem 2 is identical with that of Theorem 2 in [3], and so will be omitted.

Corollary 1. If

$$
\sum_{n=0}^{\infty} \frac{(n h(n))^{k}}{n}=O(1)
$$

and

$$
\Phi_{1}(t):=\int_{t}^{\delta} u^{-1} \phi_{1}(u) d u=O\left\{h\left(t^{-1}\right)\right\},
$$

then $\sum A_{n}(x)$ is summable $|C, 1|_{k}, k \geq 1$.

Proof. with each $p_{n}=1$, all of the conditions of Theorem 1 are satisfied.

Corollary 2. If (1.6) and (1.4) hold, then the conjugate series is summable $|C, 1|_{k}$, $k \geq 1$.

\section{References}

[1] G. S. Pandey, Multipliers for $|C, 1|$ summability of Fourier seris, Pacific J. Math. 54(1978), 177-182.

[2] B. E. Rhoades, Inclusion theorems for absolute matrix summability, J. Math. Anal. Appl. 238(1999), 82-90.

[3] W. T. Sulaiman, On absolute Nörlund summability of Fourier series and the derived Fourier series, Tamkang J. Math. 21(1990), 79-87.

Department of Mathematics, Indiana University, Bloomington, In 47405-7106, U. S. A..

Department of Mathematics, Yüzüncü Yil University, Van, Turkey.

E-mail: rhoades@indiana.edu

E-mail: ekremsavas@yahoo.com 